\title{
Investigation of the unsteadiness of a shock-reflection interaction with time-resolved particle image velocimetry
}

\author{
B. W. van Oudheusden - A. J. P. Jöbsis · F. Scarano • \\ L. J. Souverein
}

Received: 16 April 2010 / Revised: 10 September 2010 / Accepted: 4 February 2011 / Published online: 24 February 2011

(C) The Author(s) 2011. This article is published with open access at Springerlink.com

\begin{abstract}
The spatio-temporal dynamics of an impinging shock/boundary layer interaction at Mach 2 and under incipient separation conditions, has been investigated experimentally by means of high-speed particle image velocimetry (PIV). The available PIV acquisition rate of up to $20 \mathrm{kHz}$ permits a time-resolved characterization of the interaction. The dynamics of different flow regions-notably the separation region and the reflected shock-were quantified by means of temporal auto-correlation fields and pseudo-spectral analysis. The PIV data further enable to investigate the relationship between spatially extended flow features, such as shock position and bubble size, as well as the influence of the upstream boundary layer. The results confirm earlier studies that there is an important upstream effect on the present incipient interaction.
\end{abstract}

Keywords Shock wave boundary layer interaction . Particle image velocimetry

\section{Introduction}

Within the technology domain of high-speed flight, the interaction that results from the impingement of a planar

Communicated by K. Hannemann.

B. W. van Oudheusden ( $\varangle)$ · A. J. P. Jöbsis · F. Scarano ·

L. J. Souverein

Delft University of Technology, PO Box 5058,

2600 GB Delft, The Netherlands

e-mail: b.w.vanoudheusden@tudelft.nl

URL: http://www.tudelft.nl

L. J. Souverein

Institut Universitaire des Systèmes Thermiques Industriels,

Marseille, France oblique shock wave on a turbulent boundary layer has great potential impact on the vehicle performance, affecting notably the efficiency of supersonic intakes as well as being responsible for increased mechanical and thermal loads on the external structures [1]. The renewed attention for the feasibility of sustainable supersonic transport has further revived the interest in this type of shockwave boundary-layer interactions (SWBLI) in the moderate supersonic regime. In this context the European 6th framework program UFAST "Unsteady effects in shock waveinduced separation" (2005-2009) was conducted, in which the shock-reflection interaction at low supersonic Mach numbers was identified as one of the flow categories of prominent interest [2].

When of sufficient strength, the interaction displays a significant degree of unsteadiness, with a wide range of spatio-temporal scales. The observation that the reflected shock movement possesses a time scale much larger than that typical of the incoming boundary layer [1-4] has led to competing explanations of this low-frequency unsteadiness. Several researchers have proposed that it can be linked to the occurrence of extremely large-scale structures in the incoming boundary layer $[5,6]$, whereas others advocate a downstream driving mechanism, where the dynamics of the separation bubble determines the low-frequency behaviour, largely independent of upstream boundary layer conditions [7-9]. As partial reconciliation, a recent study of Souverein et al. [10] indicates that both mechanisms are likely to play a role in the interaction, concluding that the upstream effects are (more) important for weak interactions with mild or intermittent separation, whereas the downstream effects become dominant for interactions with substantial separation.

Experimental information on the temporal aspects of the interactions' unsteadiness has been provided in the past by 
high-speed qualitative visualization and point-wise timeresolved measurements from hot-wire and LDA, while the spatial instantaneous organization can be captured conveniently by particle image velocimetry (PIV). Combining the spatial and temporal information is crucial to obtain a complete description of the spatio-temporal coherence of the interaction. Recent numerical simulations using DNS $[9,11]$ or LES [12] have provided much information regarding the flow behaviour of these interactions, but remain limited in Reynolds number (which for the present experimental conditions is approximately one order of magnitude beyond the current DNS and LES simulation capabilities). Hence, extensive experimental data remain required, as means of validating numerical simulations and for exploring the high Reynolds number regime. In view of the insufficient measurement rate of conventional CCD-based PIV systems and the degraded performance of high-speed CMOS-based PIV systems (especially at the high repetition rates that would be required for supersonic flow conditions), a dual-PIV system was employed by Souverein et al. [13] to extract temporal information of an intermittently separated SWBLI at Mach 1.7 (but for otherwise similar conditions as the present experiment). In the dual-PIV approach [14] two independent CCD-based PIV-systems, separated by means of orthogonal polarization, are operated at an adjustable small time delay that is not limited by the repetition-rate restrictions of a single system. Changing the time delay over the range of interest then permits to sample the temporalcorrelation as function of time delay (which in the study ranged from 5 to $2,000 \mu \mathrm{s}$, hence, equivalent to repetition rates of $500 \mathrm{~Hz}-200 \mathrm{kHz}$ ), which gave quantitative evidence of the large variation in time scales for different flow regions.

In comparison to this previous investigation, the current study employs state-of-the-art high-speed PIV technology, which permits to obtain reliable quantitative velocity data for measurement rates up to 10 or $20 \mathrm{kHz}$. The correlation results are compared to the results of the dual-PIV approach. Due to the high acquisition rate, larger data ensembles (with corresponding smaller error of convergence) can be obtained, for time delays of $100 \mu \mathrm{s}$ and higher. Typically, ensembles of 10,000 samples were acquired ( $1 \mathrm{~s}$ of acquisition time at $10 \mathrm{kHz}$ ), whereas in the dual-PIV approach data ensembles of the order of 200 were recorded (requiring at the $5 \mathrm{~Hz}$ data acquisition rate a measurement time of $40 \mathrm{~s}$ ). In addition, the data time records, thus, obtained allow spectral information to be derived, for those phenomena for which the sampling frequency is sufficient. The spatio-temporal information provided by the high-speed PIV sequences has been further analyzed to investigate the statistical interrelationship between spatially extended flow features, notably shock position and interaction bubble size.

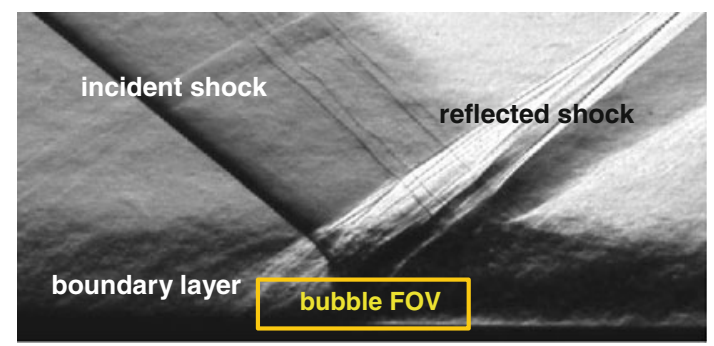

Fig. 1 Short-time-exposure schlieren visualization of the SWBLI (after [15])

\section{Experimental arrangement}

\subsection{Flow facility}

The experiments were performed in the TST-27 transonicsupersonic wind tunnel of the Delft University of Technology, with test section dimensions of $280 \mathrm{~mm}$ (width) $\times 255$ $\mathrm{mm}$ (height). The wind tunnel is operated as a blow-down facility, permitting a run time of up to $300 \mathrm{~s}$, and where the Mach number can be set by means of a continuously variable throat and flexible upper and lower nozzle walls. The test conditions for the present measurements are similar to those reported in [15]: a free stream Mach number of $2.05\left(\mathrm{U}_{\infty}=510 \mathrm{~m} / \mathrm{s}\right)$, a total temperature of $285 \mathrm{~K}$ and a stagnation pressure of 2.8 bar $(\mathrm{Re}=36 \times$ $\left.10^{6} \mathrm{~m}^{-1}\right)$.

A careful assessment of the free-stream quality was made by means of a PIV measurement at high resolution $(40 \mu \mathrm{m} / \mathrm{pixel})$ and increased pulse separation $(5 \mu \mathrm{s})$, resulting in a mean particle displacement of 65 pixels. This permitted to determine the free-stream turbulence level as being approximately $0.5 \%$.

The flow case under consideration is that of an oblique shock wave impinging on the natural turbulent boundary layer of the upper tunnel wall. The boundary layer has a thickness of about $20 \mathrm{~mm}$, with the Reynolds number based on momentum thickness being 50,000. The incident shock wave for the SWBLI is generated by a nearly full-span wedge imposing a flow deflection of $8.0^{\circ}$. The overall structure of the interaction is visualized in Fig. 1. The interaction length $\mathrm{L}$, defined as the distance between the extrapolated intersection points of the incident and reflected shocks with the wall [7], is about: $\mathrm{L} \approx 50 \mathrm{~mm} \approx 2.5 \delta$. In a previous study [15] it was verified that the interaction mean flow displayed homogeneity in the spanwise direction over an extent of at least five boundary layer thicknesses (equivalent to $30 \%$ of the test section width). As a result, the mean interaction is considered to be sufficiently two-dimensional to justify the planar PIV measurement approach applied in the present study. 


\subsection{PIV experimental arrangement}

The high-speed PIV system was set up to acquire a timeresolved characterization of the interaction at the central streamwise/wall-normal plane of the wind tunnel. The illumination and viewing configuration were similar as in [15]. The laser illumination was provided from downstream of the test section while the observation is made through the large window in the wind tunnel sidewall. The laser light sheet thickness was about $2 \mathrm{~mm}$.

Although the PIV system could be operated at recording rates up to $20 \mathrm{kHz}$, it was found that the results at $20 \mathrm{kHz}$ were deteriorated due to irregularities in the pulse separation, which introduced an additional uncertainty on the velocity measurement of about $3 \%$. Therefore, although the $20 \mathrm{kHz}$ data could still be used for visualization and correlation purposes, most of the unsteady flow analysis was carried out with the more reliable data that were obtained at $10 \mathrm{kHz}$. The data acquisition timing, including the time separation between the laser pulses, was controlled by a LaVision High Speed Controller, while data acquisition and post-processing was performed by LaVision DaVis 7.2 software.

Flow seeding was introduced in the settling chamber upstream of the test section, using either DEHS liquid seeding or the more refractive $\mathrm{TiO} 2$ solid seeding, the latter being required in particular for the experiments at the highest recording rates in view of the decreased laser power per pulse. Both seeding types have been assessed to display a particle relaxation time $\tau$ of $2-2.5 \mu \mathrm{s}$ [16]. The corresponding Stokes number $\left(\tau U_{\infty} / \delta=0.05\right)$ is sufficient to ensure a proper tracking of the turbulent fluctuations [17].

The illumination was provided by a Quantronix DarwinDuo diode-pumped dual oscillator single head Nd:YLF laser emitting at a wavelength of $527 \mathrm{~nm}$. The maximum average power of the laser at a frequency of $1-3 \mathrm{kHz}$ is $75-80 \mathrm{~W}$. At $1 \mathrm{kHz}$ the pulse energy is $25 \mathrm{~mJ}$ and the pulse duration $150 \mathrm{~ns}$; at $10 \mathrm{kHz}$ the pulse energy is $2.5 \mathrm{~mJ}$ and pulse duration is $500 \mathrm{~ns}$ (according to manufacturer specifications). The pulse separation was normally set to $3 \mu \mathrm{s}$, resulting in a particle displacement in the free stream of $1.5 \mathrm{~mm}$ between subsequent images, which corresponds to 20-40 pixels depending on magnification.

The particle images were recorded using Photron FASTCAM-SA1 1,024 × 1,024 pixel CMOS camera(s) (12bit). The maximum image recording rate of the camera at full frame resolution is $5.4 \mathrm{kHz}$, which is equivalent to a velocity data rate of $2.7 \mathrm{kHz}$, as two subsequent images are required to compute a velocity field. Increased recording rates can be achieved at a reduced frame size, up to a $40 \mathrm{kHz}$ image frame rate (permitting a $20 \mathrm{kHz}$ velocity data rate) at a $512 \times 256$ pixels frame size. Where in the present discussion reference is made to the data rate, this is always meant to imply the rate
Table 1 PIV viewing configurations

\begin{tabular}{llllll}
\hline Configuration & \multicolumn{2}{l}{ FOV size } & & \multicolumn{2}{l}{ Resolution } \\
\cline { 2 - 3 } \cline { 5 - 6 } \cline { 5 - 6 } & $(\mathrm{mm})$ & $($ pixels $)$ & & $(\mu \mathrm{m} /$ pixel $)$ & $(\mathrm{mm} /$ vector $)$ \\
\hline Overview A & $65 \times 31$ & $768 \times 384$ & & 85 & 0.68 \\
Overview B $^{\mathrm{a}}$ & $60 \times 30$ & $970 \times 512$ & & 62 & 0.50 \\
Overview C $^{\mathrm{a}, \mathrm{b}}$ & $75 \times 35$ & $1937 \times 1024$ & & 39 & 0.32 \\
Bubble $^{\mathrm{a}}$ & $56 \times 14$ & $1400 \times 384$ & & 42 & 0.34 \\
\hline
\end{tabular}

${ }^{a}$ Indicates the use of 2 cameras in side-by-side configuration

${ }^{\mathrm{b}}$ Full-frame camera sensor operation, only for up to $1 \mathrm{kHz}$

at which the velocity fields are acquired, which is, hence, half of the framing rate of the camera.

Lenses of $105 \mathrm{~mm}$ focal length were used with aperture set to 2.8 to maximize the amount of light captured by the sensor. The peak-locking effect induced by this large aperture was mitigated by slightly defocusing the imager, increasing the imaged particle size to about $2-3$ pixels. From direct inspection of the digital images we find a particles' peak intensity of 100-200 counts. The noise level of the camera was for the highest frame rate approximately 30 counts.

In some of the experiments two cameras were used, in a side-by-side configuration with a small overlap, to enhance the field of view size or to improve spatial resolution.

Pre-processing of the recorded particle images was performed with the DaVis software, using intensity normalization with the local pixel average density and min-max filtering [18]. Subsequently, an iterative multi-step cross-correlation was applied, with a final interrogation window size of $32 \times 32$ pixels and $75 \%$ overlap. The resulting vector data grid has a spacing between 0.3 and $0.7 \mathrm{~mm}$, depending on the specific FOV settings (see Table 1). The actual spatial resolution is related to the interrogation window size, however, the modern image interrogation techniques with iterative deformation have been shown to possess a more favourable spatial response than a simple "top-hat" filter [19]. Following these theoretical studies, the effective spatial resolution of the present study is estimated at half the interrogation window size, which corresponds to $0.6(0.03 \delta)$ or $1.4 \mathrm{~mm}(0.07 \delta)$, respectively.

Spurious vectors were removed by means of a median filter and substituted by interpolation. Typical cross-correlation accuracy was 0.2 pixel (r.m.s.), corresponding to a velocity accuracy of $0.5-1 \%$ of the free stream value.

Characteristic parameters for the most relevant experimental configurations (used for the acquisitions at $10 \mathrm{kHz}$ unless specified otherwise) are summarized in Table 1. As an illustration, the approximate extent and location of the "bubble FOV" are indicated in the schlieren visualization of Fig. 1. 

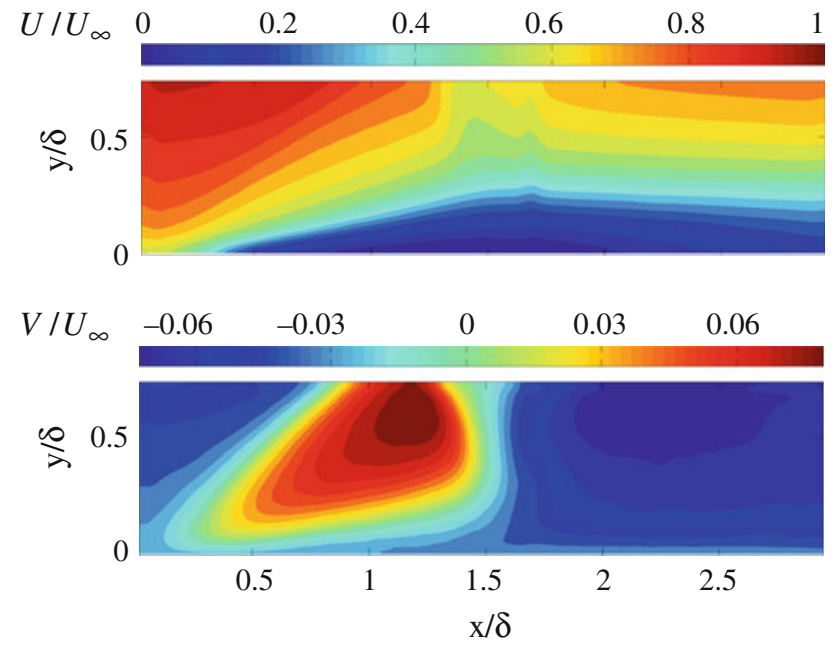

Fig. 2 Mean-flow characterization; top $U / U_{\infty}$, bottom $V / U_{\infty}$

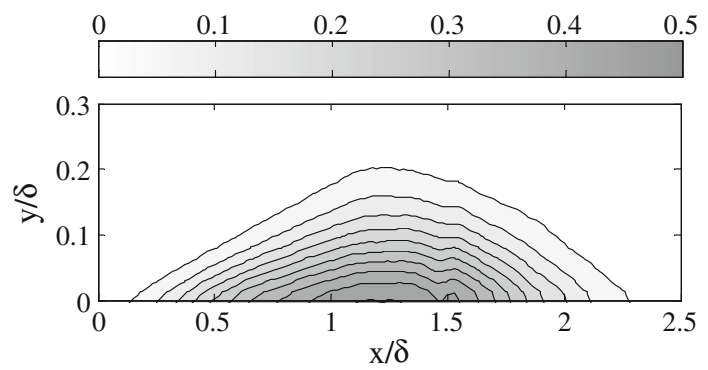

Fig. 3 Flow reversal probability (note enlarged vertical scale)

\section{Overall interaction characterization}

To characterize the overall flow field of the interaction, the distributions of the time-average longitudinal and transverse velocity components are depicted in Fig. 2, for the "bubble FOV". It may be remarked that, although no flow reversal was determined on the mean (at the present resolution), substantial backflow could be observed in a significant number of the instantaneous flow fields. Typically, reversed flow was completely absent in only $10 \%$ of the realizations. The spatial distribution of the flow-reversal probability is shown in Fig. 3 (note elongated vertical scale), and displays a maximum of about $50 \%$ near the wall at the longitudinal location of $1.3 \delta$, in the selected coordinate frame of reference as indicated. In this sense, the present interaction is considered to be one of intermittent separation, and being either marginally attached or separated on the mean (cf. [10] and [15]). The small vertical artifact in Figs. 2 and 3 near $\mathrm{x} / \delta=1.5$ results from the stitching of the images from the two cameras employed in this viewing configuration.

Figure 4 further provides a statistical characterization of the interaction activity in terms of the local turbulence levels, revealing that the $u$-fluctuation is strongest in the bubble region, while the $v$-fluctuation is most prominent around the

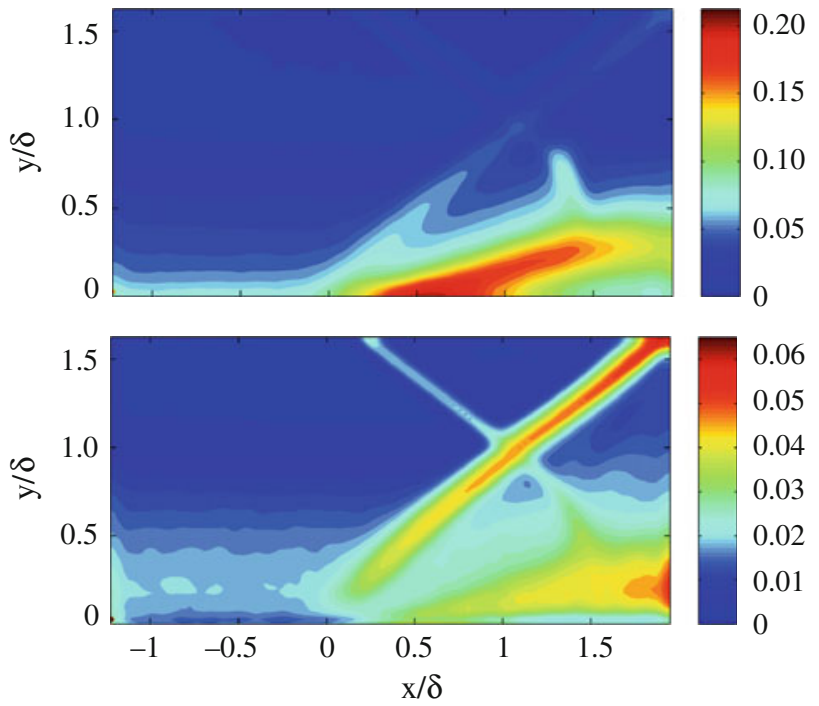

Fig. 4 Turbulence levels in the interaction (overview FOV); top $u^{\prime} / U_{\infty}$ bottom $v^{\prime} / U_{\infty}$

reflected shock and in the region downstream of the bubble, the latter reflecting the development of large-scale vortex shedding in the detached shear layer, as will be further illustrated in the next section.

The employed PIV data acquisition rates of up to $20 \mathrm{kHz}$ permit to obtain a quantitative time-resolved visualization of the interaction flow field. However, for reasons of data accuracy as stated previously, the $10 \mathrm{kHz}$ data will form the basis for the unsteady flow analysis.

Previous hot-wire measurements [20], taken at $y / \delta=0.2$ in the upstream boundary layer, revealed energetic scales in the $10 \mathrm{kHz}$ range. To investigate if these spatial scales were resolved by the current PIV measurements, Taylor's hypothesis was used to artificially increase the temporal sampling rate of the PIV measurements. This approach to transfer spatial to temporal information is justified under these conditions, where the temporal fluctuation at a stationary location is dominated by the convection of the turbulent structures. By means of a velocity-pattern correlation procedure between subsequent velocity fields, the spatial information was used to interpolate the velocity signal temporal variation at intermediate time instants. When implementing this procedure at $y / \delta=0.2$, a temporal super-sampling with a factor of 90 resulted, providing an equivalent sampling frequency of $900 \mathrm{kHz}$. Assuming that the interrogation window size acts as a low-pass filter, the equivalent cut-off frequency is about $200 \mathrm{kHz}$ (the overlap factor being 75\%). The resulting spectrum was found to show good accordance with the hot-wire results.

To illustrate the interaction dynamics, in particular that of the separated flow region covered by the "bubble FOV", a sample sequence of nine subsequent velocity vector fields 
Fig. 5 Sequence of acquisitions (at $10 \mathrm{kHz}$ rate) for streamwise velocity component (left) and corresponding vortex detector (right) reveal a substantial life-time of the separated flow region
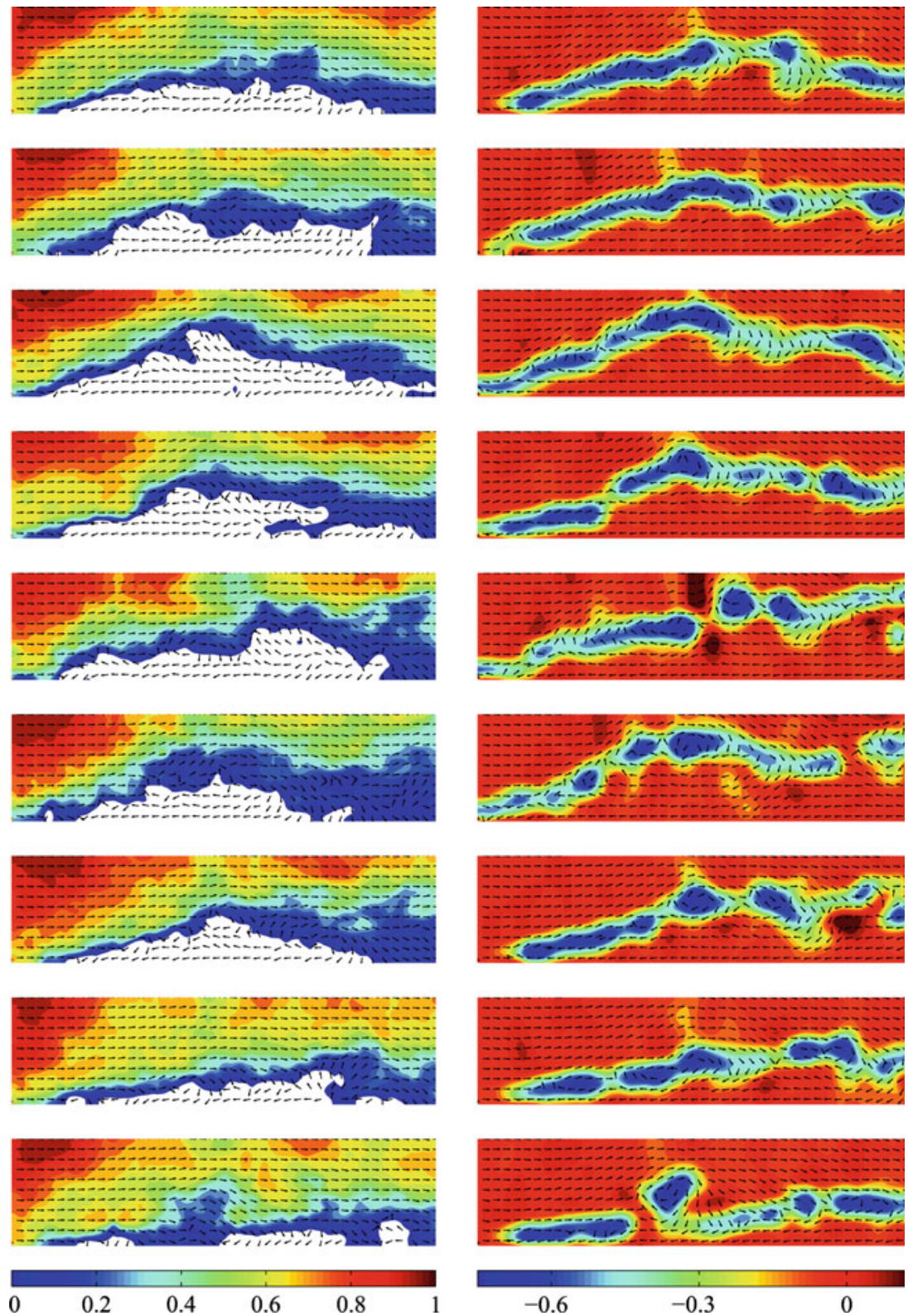

acquired at $10 \mathrm{kHz}$ rate is given in Fig. 5. In the diagrams on the left, the background colour codes the streamwise velocity component (white indicates reversed flow). The corresponding diagrams on the right characterize the accompanying shear-layer development and vortex shedding process, visualized by the vortex detector as described by Graftieaux et al [21]. This particular vortex detector employs a nonlocal criterion, which makes it more suitable for detecting large-scale vortical flow structures than local criteria based on velocity gradients, like vorticity or swirling strength. The successful application in similar shock-interaction studies based on PIV was reported in [8] and [10]. For more precise details the reader is referred to the original reference [21] or the preceding studies $[8,10]$.

The shear layer is seen to develop along the interface between the high-speed and low-speed regions, and the formation of large-scale vortices becomes evident, especially downstream of the crest of the bubble, which agrees with uncorrelated instantaneous observations from lowrepetition-rate PIV $[10,15,20]$. The time sequence gives evidence of a substantial life time of the separated flow region. From the observation that it persists over several consecutive frames, the characteristic frequency of the overall bubble dynamics can be inferred to lie near or below $1 \mathrm{kHz}$. In the 
downstream vortex-shedding region, vortices are observed to convect between subsequent images over approximately one quarter of the longitudinal size of the observation domain (hence, $15 \mathrm{~mm}$ ), corresponding to a convective velocity of about $150 \mathrm{~m} / \mathrm{s}$ (30\% of the free stream velocity). From these values an Eulerian time scale of the order of $100 \mu$ s follows (corresponding frequency $10 \mathrm{kHz}$ ). The associated Strouhal number, based on the interaction length $L$, is of order unity, which agrees reasonably well with values reported in [7], that were derived from wall-pressure signals.

\subsection{Shock unsteadiness}

The transverse turbulence component field in particular (Fig. 4) contains clear traces of the reflected and (albeit to a lesser extent) the incident shock, as a result of the prominent change in the $v$-component over the shocks. Time records of this velocity component can, hence, be employed as representative indicators for the shock motion. The $v$-component is favoured as shock-motion indicator over the $u$-component, as it reflects the flow deflection which is usually easier to identify than the flow-magnitude change associated to compression.

The shock activity in three distinctly different flow regions has been assessed in this manner, viz. the incident shock, the reflected shock and the reflected shock foot. The shock foot is defined here as the region of the reflected shock system inside the boundary layer. The corresponding "probe locations" were taken at $y / \delta=1.32$ for both the incident and reflected shock, and at $y / \delta=0.64$ for the shock foot. The longitudinal position of each of the probe locations corresponds to that of the local maximum in the $v$-component r.m.s. (incident shock: $\mathrm{x} / \delta=0.63$; shock foot: $\mathrm{x} / \delta=0.67$; reflected shock: $\mathrm{x} / \delta=1.52$ ). A sample time trace of $25 \mathrm{~ms}$ duration (out of the total measurement time of about $1 \mathrm{~s}$ ) obtained at a sampling rate of $10 \mathrm{kHz}$, is depicted in Fig. 6 . The velocity fluctuations at the reflected shock (red) and the shock foot (blue) show a high degree of correlation, but with additional high-frequency fluctuations for the shock foot. This observation supports the idea that the shock foot displays a combination of the relatively slow motion of the shock system as a whole, in (partial) response to the bubble dynamics, and an additional high-frequency response towards the passing of turbulent structures from the incoming boundary layer. The time trace of the incident shock, on the other hand, shows an appreciable smaller dynamics, in particular, the large-scale low-frequency behaviour being absent suggests that there is little correlation between the incident shock and the large-scale interaction dynamics.

The level of correlation of the different regions of the flow with respect to the reflected shock motion was assessed by correlating the $v$-component at the reference point (representing the shock motion) with the velocity fluctuations
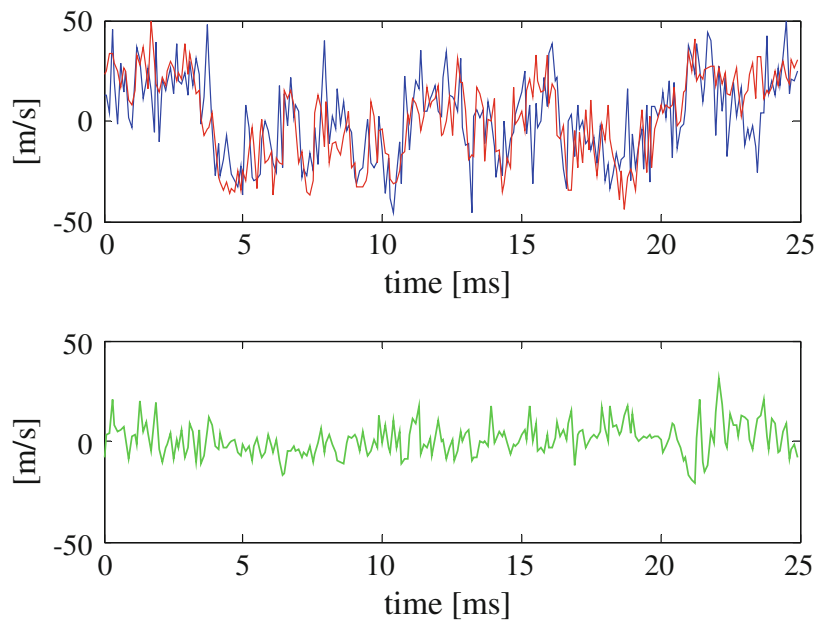

Fig. 6 Time records for $v$-velocity at shock probe locations: red reflected shock; blue shock foot; green incident shock

everywhere else in the flow field. The reason why the change in the $v$-component at the reference point can be related to the shock motion is that across the reflected shock the flow is deflected upwards, leading to a positive gradient of $v$ in the streamwise direction. Hence, the $v$-component at the reference point will decrease for a more downstream position of the shock and increase for a more upstream position. The results are expressed in terms of the (normalized) correlation coefficient:

$R_{v_{r e f}, u_{i}}(x)=\frac{\overline{u_{i}^{\prime}(x, t) \cdot v^{\prime}\left(x_{r e f}, t\right)}}{\sigma_{u_{i}}(x) \cdot \sigma_{v}\left(x_{r e f}\right)}$

Here, $u_{i}$ represents either the $u$ or $v$-component of the velocity, while $\sigma$ stands for the standard deviation of the particular variable.

Figure 7 depicts the correlation coefficient spatial distribution for the $v$-component (top) and for the $u$-component (bottom), respectively. The upper graph reveals that there is a high degree of correlation along the entire reflected shock structure, which is consistent with an overall motion of the complete shock system. Also in this representation the correlation with the incident shock is observed to be very small, the $v-v_{\text {ref }}$-correlation coefficient being less than 0.1 . The


of correlation across the reflected-shock system, but in addition substantial negative correlation levels are observed in both the upstream boundary layer $\left(R_{u v}\right.$ up to -0.25$)$ and in the downstream region $\left(R_{u v}\right.$ up to -0.33$)$. Note that a negative value of the correlation in this situation indicates that an increase of the local $u$-component is expected to be accompanied by a decrease of the $v$-component at the reference point, the latter corresponding to a downstream displacement of the reflected shock. Although the correlation is not necessarily indicative of any causal relation, it illustrates 


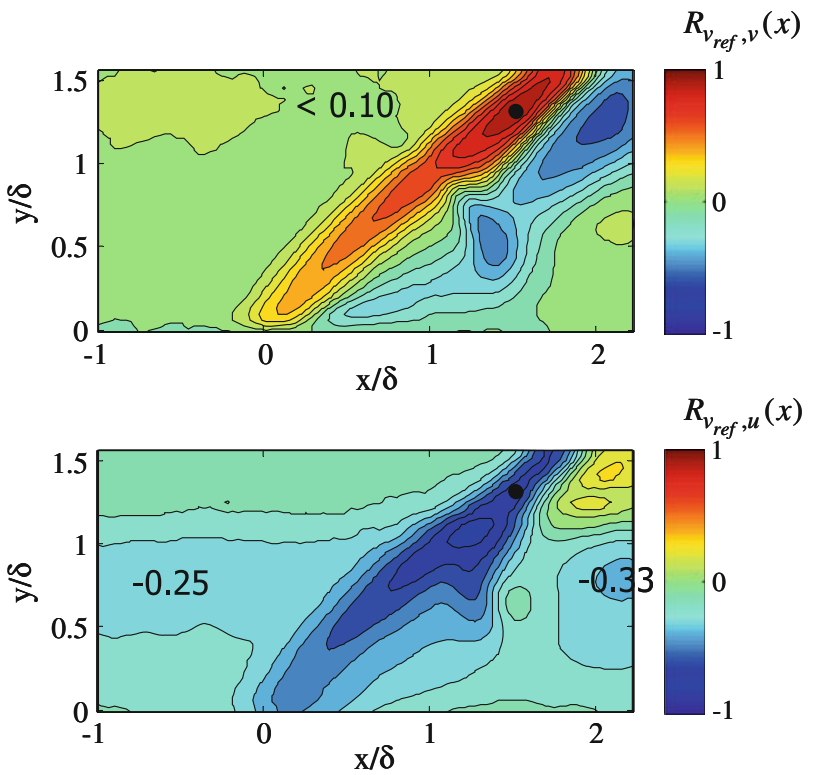

Fig. 7 Spatial distribution of the velocity correlation coefficient between the transverse velocity in the shock reference point (indicated by the black dot) and the transverse (top) or longitudinal (bottom) velocity component, respectively

that a downstream displacement of the reflected shock is statistically accompanied by an increase of the momentum in the incoming boundary layer, as well as a contraction of the low-speed bubble region.

\subsection{Shock dynamics characterization}

A spectral estimation of the velocity time traces at the three selected shock probe locations was made employing Welch's averaged modified periodogram method as implemented in the cpsd function in MatLab. To smoothen the resulting spectra, a relatively small window size of 300 samples was chosen. The resulting pseudo-spectra are shown in Fig. 8 (premultiplied, arbitrary units). The reflected-shock dynamics are observed to contain a low-frequency energetic dominance in the range of 300-400 Hz. The corresponding Strouhal number based on the interaction length $L$ is $0.03-$ 0.04 , which compares well with the values reported in other studies [7]. Comparison of the spectra further confirms the higher spectral content of the shock foot in comparison to that of the reflected shock, as observed in the time series of Fig. 6. However, as the spectrum maintains to display an increase towards the end of the spectrum (at the Nyquist frequency) it must be concluded that this part of the spectrum is unreliable, as a result of the aliasing of high (physical) frequencies present in the shock motion. For the reflected shock, on the other hand, a comparison of spectra of the shock motion obtained at different sampling rates $(5,10$ and $20 \mathrm{kHz}$ ) suggests that the shape of the spectrum presented here can be considered representative in the frequency range

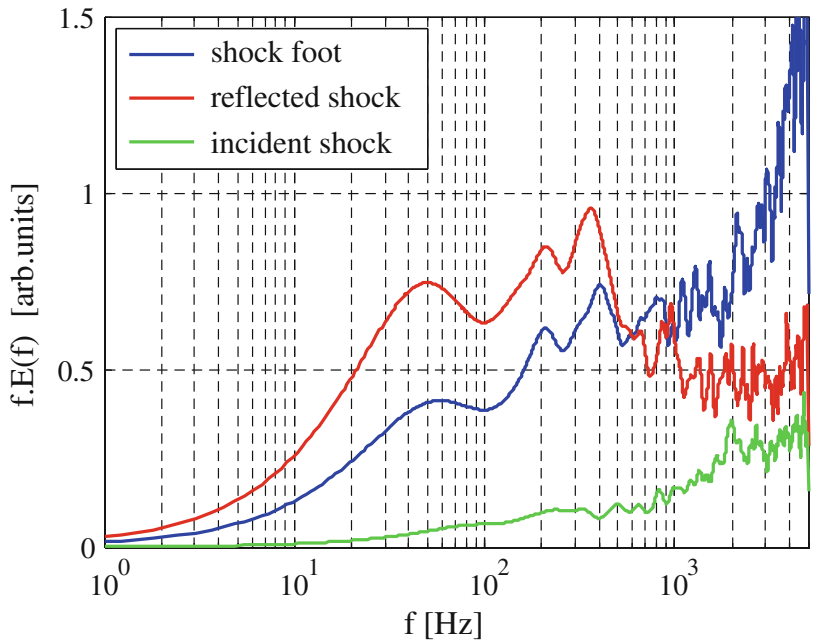

Fig. 8 Pseudo pre-multiplied power spectra for the shock motions (color legend as in Fig. 7)

up to $2 \mathrm{kHz}$. The shock-foot dynamics is hence distinct from that of the reflected shock (outside the boundary layer), with the spectrum suggesting a shift in the dominant frequency, if any, towards values in the order of $1 \mathrm{kHz}$. Hot-wire measurements carried out in this region at $\mathrm{y} / \delta=0.2$ revealed a spectral maximum for $1-3 \mathrm{kHz}$ [20], corresponding to a Strouhal number of about $0.1-0.3$. The results hint that there may be spectral content at higher frequencies, which cannot properly be resolved, however, due to sampling frequency limitations.

\subsection{Temporal-correlation analysis}

A subsequent temporal-correlation analysis was carried out to obtain a characterization of the different time-scales of the flow field that is not distorted by the sampling-frequency limitations that affect the spectra. Following the approach of Souverein et al. [13], the spatial distribution of the temporal auto-correlation of the streamwise velocity component:

$R_{u u}(x, \Delta t)=\frac{\overline{u^{\prime}(x, t) \cdot u^{\prime}(x, t+\Delta t)}}{\sigma_{u}^{2}(x)}$

was evaluated for different values of the time delay $\Delta t$. In view of the data acquisition rate of $10 \mathrm{kHz}$, results are available for time delays of integer multiples of $100 \mu \mathrm{s}$ (which is about $3 \delta / U_{\infty}$ ). Figure 9 shows two distinguishing examples, for 100 and $500 \mu \mathrm{s}$, clearly revealing the relatively large time scale of the reflected shock region. The bubble region, while still being significantly correlated at $100 \mu \mathrm{s}$, has no discernible correlation any more at the higher time delay of $500 \mu \mathrm{s}$.

For selected flow regions averaged values of the correlation have been determined, focussing on the reflected shock region (outside the boundary layer), the shock foot and the shear layer region immediately above the bubble. A more 

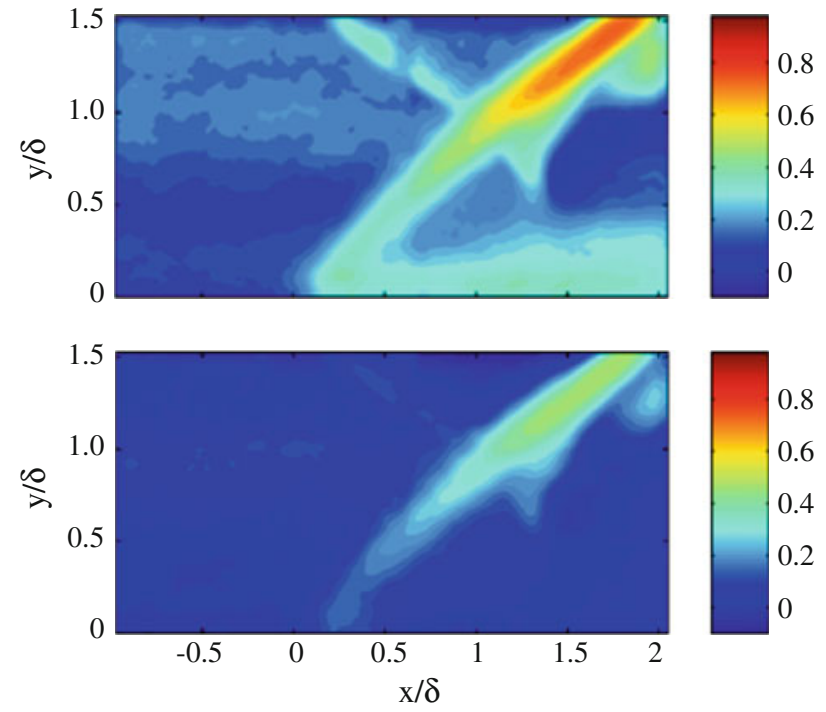

Fig. 9 Temporal auto-correlation coefficient map of the streamwise

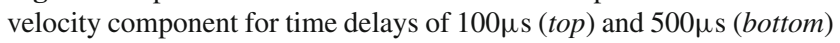

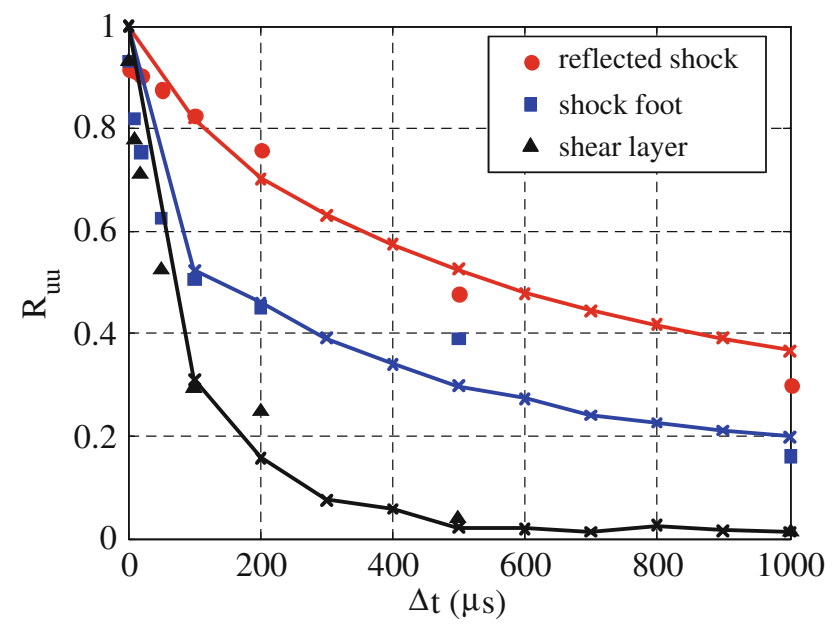

Fig. 10 Temporal auto-correlation coefficient as function of time delay, for selected regions of the interaction. Filled symbols indicate dual-PIV results from Souverein et al. [13]

precise definition of the extent of the different regions can be found in [13]. The results are displayed in Fig. 10, where they have been compared to the dual-PIV data from [13], which were obtained in the same facility at very similar interaction conditions. The two experimental approaches show a good agreement. The relatively slow decay of the autocorrelation for the reflected shock corresponds to the lowfrequency behaviour observed in the spectra. The shock foot curve has the character of a multiple-time scale phenomenon, with a relatively rapid initial decrease (which cannot be captured in detail in the present experiment, but is confirmed by the dual-PIV results) and a slowly decaying tail. This would correspond to high and low-frequency content of the spectrum, respectively. These observations would support the previous remarks about the dual response nature of the shock foot, as inferred from the time trace of Fig. 6.

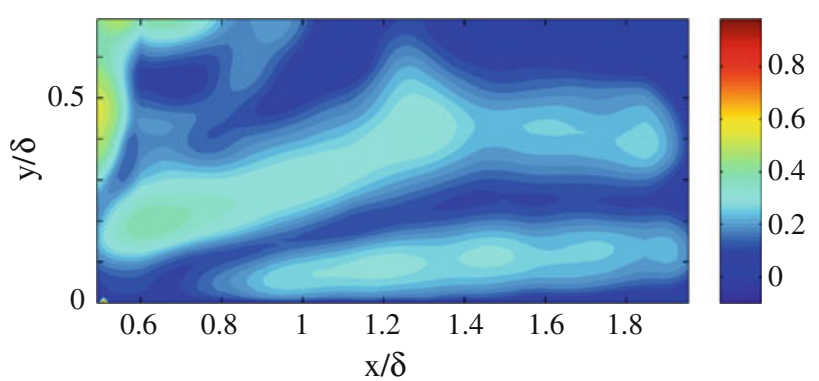

Fig. 11 Temporal auto-correlation coefficient map for the vortex detector (time delay $100 \mu \mathrm{s}$ )

The shear layer region displays a substantially smaller time scale than the reflected shock. However, this value is not representative of the time scale of the bubble itself, which as will be shown later in Sect. 4.1, is about one order higher. This seeming discrepancy is explained by the fact that the autocorrelation of the velocity fluctuations for the shear layer region is dominated by the vortex shedding, which occurs at a significantly higher frequency than the overall bubble motion. The observed time correlation is in reasonable agreement with the Eulerian time scale of $100 \mu$ s that was estimated from the vortex convection in connection to the visualization in Fig. 5.

Figure 11 displays the correlation for the vortex detector at a time delay of $100 \mu \mathrm{s}$, for a magnified field of view. Two separate regions of increased correlation are clearly visible, corresponding to large and small bubbles, respectively (compare Fig. 5). This supports the concept of an intermittent behavior between separated and attached flow, with relatively rapid transitions in between.

\section{Dynamics of spatial interaction features}

In addition to local flow field properties (such as velocity), the whole-field capability of PIV also permits a temporal characterization of spatially extended features, such as shock position or separation region size.

To investigate the shock dynamics, instantaneous shock positions were determined in each of the individual realizations. Also in these procedures, for the reasons mentioned previously, the $v$-component was used as a suitable indicator of flow deflection associated with shocks. For the reflected shock, outside the boundary layer, this was performed by matching the time-average streamwise $v$-profile to the instantaneous one, by shifting it to agree at the position of the strongest positive gradient (i.e., upward flow deflection). Instead, for the reflected shock foot, inside the boundary layer, the shock position is taken directly as the location of the maximum streamwise gradient of the $v$-component. To suppress noise peaks in the instantaneous $v$-distribution in the latter case, a linear smoothing over 20 vectors in streamwise 
(a) reflected shock position

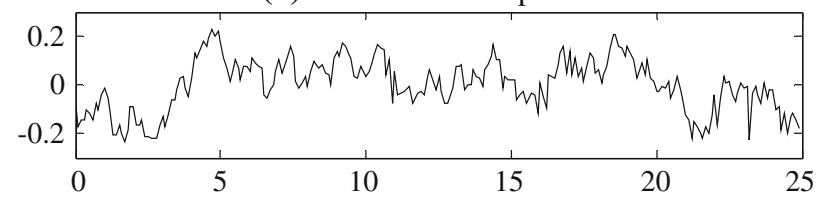

(b) shock foot position

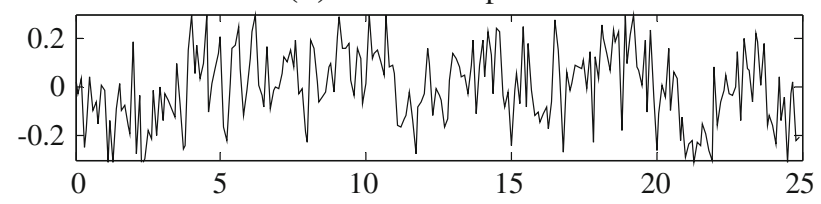

(c) bubble size

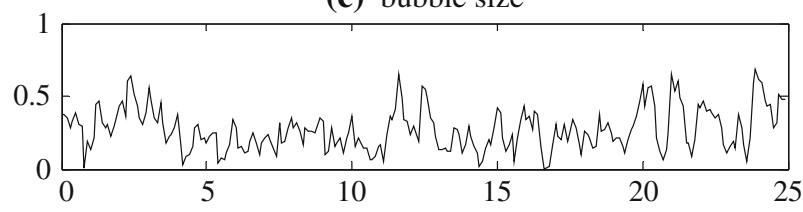

(d) boundary layer momentum

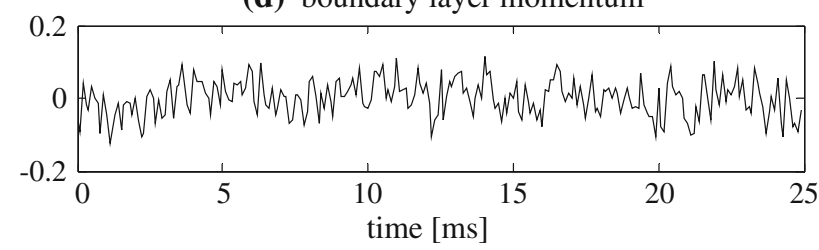

Fig. 12 Time records for shock positions, bubble size and boundary layer momentum

direction was applied, which does not essentially affect the shock location determination.

Regarding the interaction bubble, it has been remarked previously that the interaction displays a character of incipient separation, with no (or marginal) flow reversal on the mean. The separation and occurrence of backflow is hence highly intermittent. Although no flow reversal is present in only $10 \%$ of the realizations, the cross-sectional area of the flow-reversal region displays a highly skewed distribution, with an average size of about $0.07 \cdot \delta^{2}$. In addition, as can be observed from the visualizations of Fig. 5, the detached shear layer can be associated with an interface at a positive value of the streamwise velocity. Hence, the ("separation") bubble was interpreted more broadly in the analysis as the low-speed region where the velocity is below a certain threshold value. The threshold has been taken here as $90 \mathrm{~m} / \mathrm{s}$ (18\% of the free stream value). It was verified that the results were not critically sensitive to this value. For this particular choice, the average cross-sectional size of the bubble was $0.28 \cdot \delta^{2}$.

\subsection{Time records, frequency spectra and temporal-correlation}

Figure 12 displays an example time record of 250 samples recorded at $10 \mathrm{kHz}$ rate, for the reflected-shock and shock-foot motion relative to their mean position (both scaled
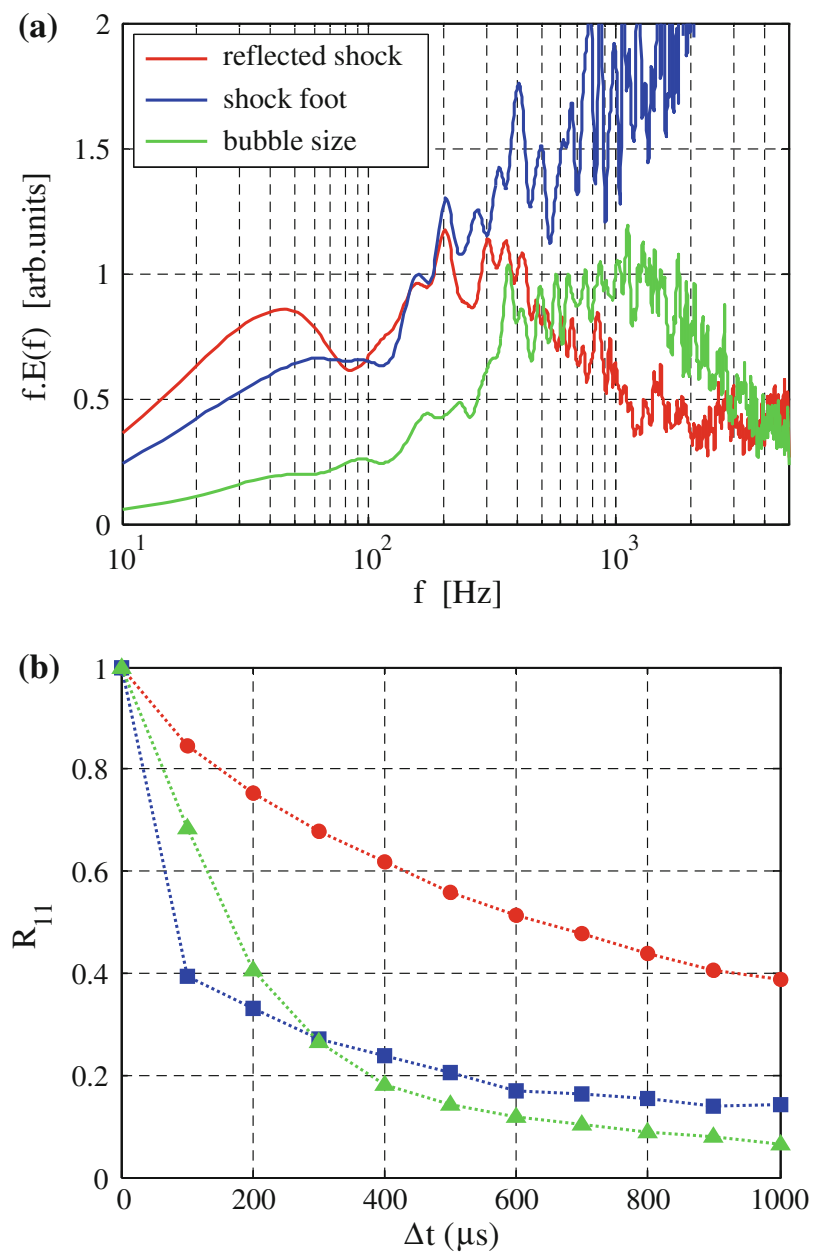

Fig. 13 Temporal characterization of the interaction region: shock positions and bubble size (a), pseudo pre-multiplied power spectra (arbitrary units) (b), temporal auto-correlation coefficient as function of time delay

with $\delta$ ), the bubble size (the observed cross-sectional area scaled with an area the size of $\delta \times \delta$ ) and the relative change of the boundary layer average momentum (evaluated over an area of approximately $0.5 \delta \times 0.5 \delta$, immediately upstream of the interaction). Directly obvious is the relative low-frequency content of the reflected shock motion and the bubble size variation with respect to the other two phenomena.

The spectral characterization of these properties of the interaction region is depicted in Fig. 13 in terms of the (premultiplied) pseudo-spectra and of the temporal correlation coefficient, for the reflected shock and shock foot positions and for the bubble size. Similar as for the velocity correlation, see (2), the auto-correlation $R_{11}(\Delta t)$ or cross-correlation $R_{12}(\Delta t)$ of the spatial features under study were computed as function of time delay, according to:

$$
R_{i j}(\Delta t)=\frac{\overline{\phi_{i}^{\prime}(t) \cdot \phi_{j}^{\prime}(t+\Delta t)}}{\sigma_{i} \cdot \sigma_{j}}
$$


where $\phi$ represents any of the interaction features considered.

For both the reflected shock and the shock foot the results are very similar to the analysis of the velocity fluctuations, see Fig. 8 for the spectra and Fig. 9 for the time-correlation. The spectrum for the bubble size displays a maximum in a frequency range around $1 \mathrm{kHz}$, which is distinctly higher than for the reflected shock. The corresponding Strouhal number based on the interaction length $\mathrm{L}$, amounts to 0.10 . Also the time-correlation results display a faster decay for the bubble size than for the reflected shock, which supports the difference in dominant frequency observed in the spectra.

\subsection{Cross-correlation and coherence}

The extent of correlation between different spatial phenomena was investigated by means of conditional averaging, temporal cross-correlation and spectral coherence analysis.

\subsubsection{Conditional averaging results}

Based on a specific conditional averaging criterion (like shock position or bubble region size), subsets of about 600 vector fields were extracted to be representative of small and large values of the criterion. Each set corresponds to $6 \%$ of the total ensemble of about 10,000 for a specific experiment. In this selection, the most extreme values were discarded to avoid that rare and possibly erroneous events would contaminate the conditional-averaging results. Averaging the flow fields of each of these subsets then provided the conditionally-averaged flow fields corresponding to the "extreme events", of either large or small values of the conditioning criterion. To highlight the effect of the particular conditioning criterion, the difference between the two extreme situations was evaluated, by subtracting the "lowvalue flow field" from the "high-value flow field". Figure 14 displays the corresponding differential velocity distributions (for the $u$-component) for the most relevant conditioning criteria investigated. The resulting plots have, hence, to be interpreted as representing the change in the streamwise velocity when the conditioning parameter changes from a small value to a large value. Consequently, a positive value indicates that the $u$-velocity at that location increases with the value of the conditioning parameter (and vice versa). In addition to the cases reported here, the conditional-averaging analysis confirmed that free stream velocity and incident shock position (which showed to be connected) do not have a noticeable impact on the interaction, hence ruling out incident shock fluctuations as a driving mechanism for the unsteadiness of the interaction.

Conditioning on the reflected-shock position (Fig. 14-top) reveals a strong link with the fluctuations in the bubble size: with the shock in the most upstream position the bubble region is largest, with reversed flow on the mean. Also,
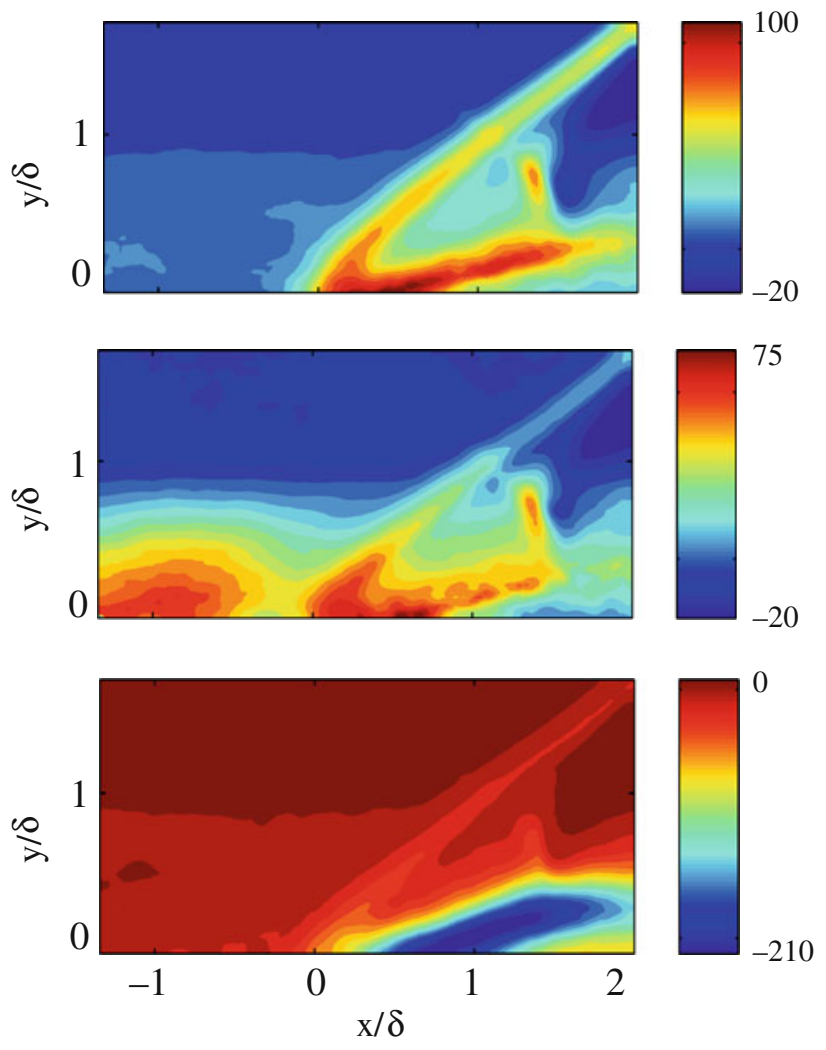

Fig. 14 Conditional-averaging results: differential $u$-velocity distributions between "large" and "small" criterion events. Condition criterion: reflected shock position (top); boundary layer velocity (centre); bubble size (bottom)

the conditional averaging reveals a (weak) relation with the upstream boundary layer: a more downstream position of the shock is associated with a higher streamwise velocity in the boundary layer, in accordance with the velocity correlation results depicted in Fig. 7. A similar dependence between shock motion and the incoming boundary layer was observed by Beresh et al. [23] for a compression ramp interaction. However, the velocity variation in the boundary layer is approximately a factor of 5 smaller than that in the bubble region.

When conditioning on the average boundary layer streamwise velocity (Fig. 14-centre), the link between boundary layer and the bubble is much stronger (the variation in both regions being of the same order), while the impact on the reflected shock, although still noticeable, is smaller than in the previous case. Conversely, when conditioning on the bubble size (Fig. 14-bottom), very little connection with in the incoming boundary layer velocity is detected, while the correlation with the reflected shock position is again clear.

In conclusions, the conditional-averaging results seem to indicate that there is indeed a strong and immediate connection between the bubble size and the reflected shock position. There is evidence for an effect of the incoming boundary 


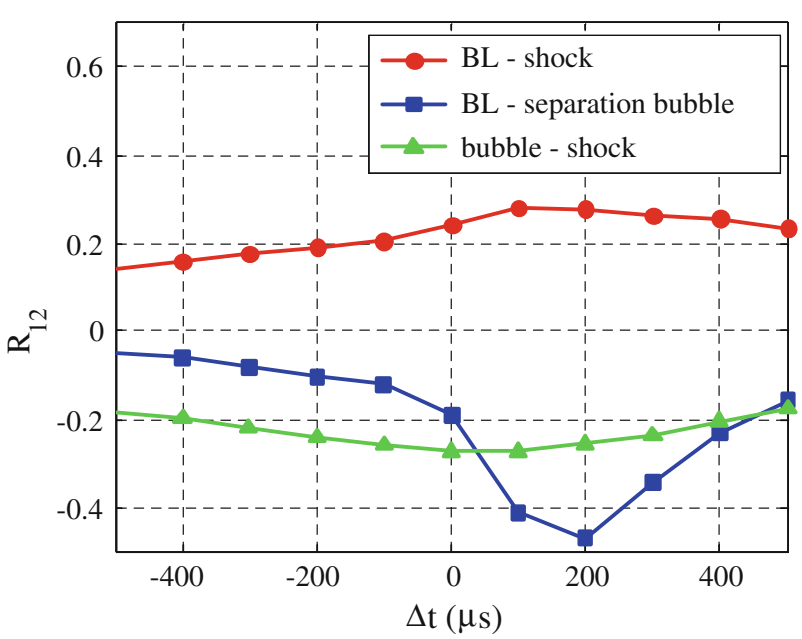

Fig. 15 Temporal cross-correlations between boundary layer momentum, shock position and bubble size

Table 2 Cross-correlation results

\begin{tabular}{llcl}
\hline Phenom. 1 & Phenom. 2 & Max. $\mathrm{R}_{12}$ & Delay $1 \rightarrow 2(\mu \mathrm{s})$ \\
\hline Shock foot & Reflected shock & 0.70 & 50 \\
Reflected shock & Bubble size & -0.28 & 0 \\
Shock foot & Bubble size & -0.36 & 100 \\
BL momentum & Reflected shock & 0.26 & 100 \\
BL momentum & Shock foot & 0.23 & $0-50$ \\
BL momentum & Bubble size & -0.50 & 150
\end{tabular}

Reflected shock and shock foot refer to their position; BL momentum refers to the average velocity in the lower half of the boundary layer (not taking variable density into account)

layer momentum, primarily through its effect on the bubble. However, although high/low speed in the boundary layer is found to correspond to a similar change in velocity in the bubble, the opposite conclusion is not supported: apparently a large/small bubble size is to a lesser degree linked to the instantaneous velocity in the incoming boundary layer. A plausible explanation for this could be an attenuated response of the bubble to the inflow conditions.

\subsubsection{Cross-correlation results}

As the conditional averaging is applied to instantaneous realizations it does not take the effect of a possible time delay into account. Therefore, a further quantification of the observed coherence between the different flow region phenomena was made by computing their temporal cross-correlation $R_{12}(\Delta t)$ as function of time delay. Selected cross-correlation curves as function of time delay are depicted in Fig. 15, while Table 2 lists the maximum value of the cross-correlation coefficients, which usually occurred at a time delay at or near zero (in the latter case the temporal offset could usually be explained from a convective delay). Note that in view of

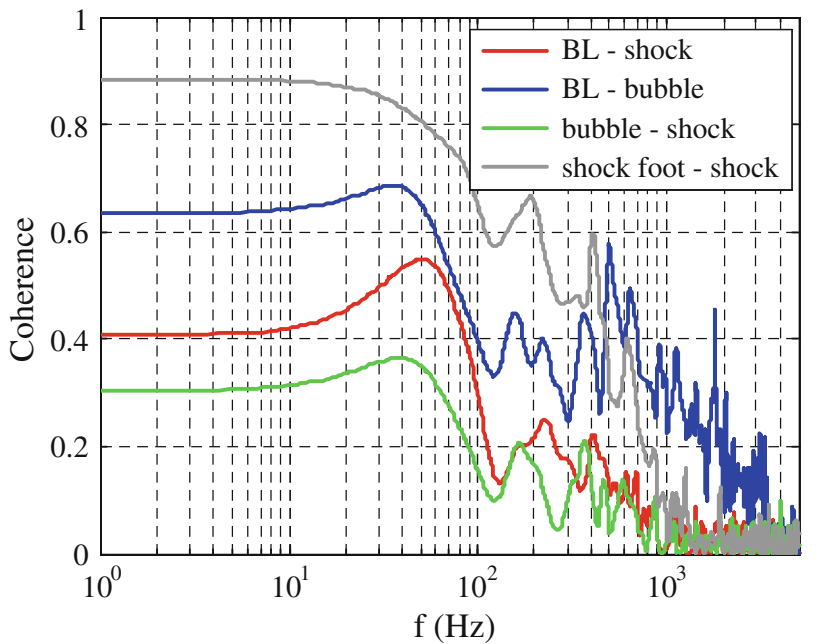

Fig. 16 Coherence between boundary layer momentum, shock position and bubble size

the data acquisition rate of $10 \mathrm{kHz}$, only an estimate of the time delay between the two phenomena could be made, with a precision of the order of at best $50 \mu \mathrm{s}$.

The first entry of Table 2 shows a high correlation between the reflected shock and the shock foot, implying that on overall the shock system moves as a whole. The negative correlation between the shocks and bubble means that a downstream shock position is accompanied by a reduction in bubble size, in agreement with the conditional-averaging results. From the correlation it appears that the connection with the shock foot is stronger, which is not surprising as this is the part of the shock directly upstream of the low-speed bubble region.

Assessing the possible impact of the incoming boundary layer as a driving component in the interaction, it is found that the momentum in the boundary layer correlates positively with the shock position (higher momentum corresponds to a downward displacement of the shock) but that the correlation is relatively weak. Much stronger is the correlation between the boundary layer and the bubble size. The negative value indicates that higher momentum in the boundary reduces the bubble size. The variation of the cross-correlation coefficient with time delay for the latter two effects (see Fig. 15) shows especially a prominent delay for its influence on the bubble, with a maximum of the correlation being more than twice the value at zero time delay. The PIV study of Humble et al. $[6,20]$ reports values of about $0.2-0.3$ for the instantaneous correlation between boundary layer shape factor, reflected shock position and bubble size, which are very similar to the values found here for zero time delay.

\subsubsection{Coherence results}

The coherence coefficient (Fig. 16) indicates that the strong correlation between reflected shock and the shock foot is 
explained especially by their high coherence in the lowfrequency domain. From a low-frequency value of about 0.9, the coherence drops off above frequencies of the order of $100 \mathrm{~Hz}$. The boundary layer (momentum) and the bubble size maintain a substantial coherence up to the range of the dominant reflected shockwave frequency (around $300 \mathrm{~Hz}$ ). As the reflected-shock motion is concerned, a similar coherence is observed with the bubble as well as with the boundary layer momentum. In conclusion, the coherence analysis indicates that at the more energetic moderate frequencies of the reflected shock motion (between $100 \mathrm{~Hz}$ and $1 \mathrm{kHz}$ ), the boundary layer and bubble effects are of similar order, whereas a high coherence between boundary layer momentum and bubble size is maintained, which seems to support that the boundary layer has an important role in the unsteadiness of the interaction through the effect it has on the bubble.

\section{Conclusions}

The temporal dynamics of a supersonic shock wave boundary layer interaction, under intermittently separated conditions, has been investigated experimentally by means of high speed PIV. Acquisition rates of up to $20 \mathrm{kHz}$ were used to obtain a time-resolved characterization of the interaction flow field. Due to laser-pulse synchronization problems at the highest acquisition rate, most of the quantitative analysis was carried out for data acquired at $10 \mathrm{kHz}$, for which the velocity data are reliable. The reflected shock displays a typical energetic frequency that corresponds to a Strouhal number $S_{L}$ of about $0.03-0.04$, which agrees with various other studies. The bubble dynamics were found to occur at a somewhat higher frequency $\left(S_{L} \sim 0.1\right)$. The difference in time scales for the different regions in the flow was further quantified by means of the temporal auto-correlation, which were found in good agreement with the results from a previous study employing a dual-PIV approach [13]. To quantify and correlate the possible interrelation of different flow phenomena-notably the incoming boundary layer, the bubble region and the reflected shock - several data analysis techniques were applied: conditional averaging, auto-correlation and coherence. The results confirm earlier studies that for this intermittently separated interaction there appears to be a substantial upstream influence. However, both the conditional averaging and the crosscorrelation results, as well as the coherence analysis, indicate a more immediate link of the boundary layer to the bubble than to the shock itself. This observation, together with the finding that the bubble dominant frequency is intermediate between the frequency of the reflected shock and that of the shock foot (the latter likely to be representative of the frequency of excitation by the incoming boundary layer), could be in support of the concept that the bubble dynamics act as a low-pass resonance filter that determines the dominant frequency of the overall interaction and the reflected shock in particular $[7,11,24]$.

Open Access This article is distributed under the terms of the Creative Commons Attribution Noncommercial License which permits any noncommercial use, distribution, and reproduction in any medium, provided the original author(s) and source are credited.

\section{References}

1. Dolling, D.S.: Fifty years of shock-wave/boundary-layer interaction research: what next?. AIAA J. 39(8), 1517-1531 (2001)

2. Doerffer, P.: European research on unsteady effects of shock wave induced separation UFAST project. 8th ISAIF. Lyon, France (July 2007, ISAIF8-0051) (2007). http://www.lmfa.ec-lyon.fr/ISAIF8/

3. Smits, A.J., Dussauge, J.P.: Turbulent Shear Layers in Supersonic Flow, 2nd edn. Springer, New York (2006)

4. Clemens, N., Narayanaswamy, V.: Shock/Turbulent Boundary Layer Interactions: Review of Recent Work on Sources of Unsteadiness. AIAA-2009-3710, 39th AIAA Fluid Dynamics Conference. San Antonio, Texas, 22-25 June 2009

5. Ganapathisubramani, G., Clemens, N.T., Dolling, D.S.: Effects of upstream boundary layer on the unsteadiness of shock-induced separation. J. Fluid Mech. 585, 369-394 (2007)

6. Humble, R.A., Elsinga, G.E., Scarano, F., van Oudheusden, B.W.: Three-dimensional instantaneous structure of a shock wave/turbulent boundary layer interaction. J. Fluids Mech. 622:33-62 (2009). (see also: J. Fluid Mech. 635, 2009, 47-74)

7. Dussauge, J.P., Dupont, P., Debiève, J.F.: Unsteadiness in shock wave turbulent boundary layer interactions with separations. Aerosp. Sci. Techn. 10, 85-91 (2006)

8. Dupont, P., Piponniau, S., Sidorenko, A., Debiève, J.F.: Investigation by particle image velocimetry measurements of oblique shock reflection with separation. AIAA J. 46(6), 1365-1370 (2008)

9. Pirozzoli, S., Grasso, F.: Direct numerical simulation of impinging shock wave/turbulent boundary layer interaction at $\mathrm{M}=$ 2.25. Phys. Fluids 18, 065113 (2006)

10. Souverein, L.J., Dupont, P., Debiève, J.F., Dussauge, J.P., van Oudheusden, B.W., Scarano, F.: Effect of interaction strength on unsteadiness in turbulent shock-wave-induced separations. AIAA J. 48, 1480-1493 (2010)

11. Wu, M., Martin, M.P.: Analysis of shock motion in shockwave and turbulent boundary layer interaction using direct numerical simulation data. J. Fluid Mech. 594, 71-83 (2008)

12. Touber, E., Sandham, N.: Large-eddy simulation of lowfrequency unsteadiness in a turbulent shock-induced separation bubble. Theor. Comp. Fluid Dyn. 23, 79-107 (2009)

13. Souverein, L.J., van Oudheusden, B.W., Scarano, F., Dupont, P.: Application of a dual-plane particle image velocimetry (dualPIV) technique for the unsteadiness characterization of a shock wave turbulent boundary layer interaction. Meas. Sci. Tech. 20(7), 074003 (2009)

14. Kähler, C.J., Kompenhans, J.: Fundamentals of multiple plane stereo PIV. Exp. Fluids 29(Suppl), S70-S77 (2000)

15. Humble, R.A., Scarano, F., van Oudheusden, B.W.: Experimental study of an unsteady impinging shockwave/ turbulent boundary layer interaction using PIV. AIAA 2006-3361, 36th AIAA Fluid Dynamics Conference and Exhibit. San Francisco, USA 5-8 June 2006

16. Ragni, D., Schrijer, F., van Oudheusden, B.W., Scarano, F.: Particle tracer response across shocks measured by PIV. Exp. Fluids (2010) (to be published). doi:10.1007/s00348-010-0892-2

17. Samimy, M., Lele, S.K.: Motion of particles with inertia in a compressible free shear layer. Phys. Fluids A 3, 1915-1923 (1991) 
18. Raffel, M., Willert, C.E., Werely, S.T., Kompenhans, J.: Particle Image Velocimetry: A Practical Guide. Springer, Berlin (2007)

19. Schrijer, F., Scarano, F.: Effect of predictor-corrector filtering on the stability and spatial resolution of iterative PIV interrogation. Exp. Fluids 45, 927-941 (2008)

20. Humble, R.A.: Unsteady Flow Organization of a Shock Wave/ Boundary Layer Interaction. PhD Thesis, Delft University of Technology, The Netherlands (2008). http://repository.tudelft.nl/

21. Graftieaux, L., Michard, M., Grosjean, N.: Combining PIV, POD and vortex identification algorithms for the study of unsteady turbulent swirling flows. Meas. Sci. Tech. 12, 1422-1429 (2001)
22. Dupont, P., Haddad, C., Debiève, J.F.: Space and time organization in a shock-induced separated boundary layer. J. Fluid Mech. 559, 255-277 (2006)

23. Beresh, S.J., Clemens, N.T., Dolling, D.S.: Relationship between upstream turbulent boundary-layer velocity fluctuations and separation shock unsteadiness. AIAA J. 40(12), 2412-2422 (2002)

24. Piponniau, S., Dussauge, J.P., Debieve, J.F., Dupont, P.: A simple model for low-frequency unsteadiness in shock-induced separation. J. Fluid Mech. 629, 87-108 (2009) 